\title{
ON THE SUPPORT OF SYMMETRIC INFINITELY DIVISIBLE AND STABLE PROBABILITY MEASURES ON LCTVS
}

\author{
BALRAM S. RAJPUT
}

\begin{abstract}
It is shown that the topological support (supp.) of a $\tau$-regular, symmetric, infinitely divisible (resp. stable of any index $\alpha \in(0,2)$ ) probability measure on a Hausdorff LCTVS $E$ is a subgroup (resp. a subspace) of $E$. The part regarding the support of a stable probability measure of this theorem completes a result of A. De-Acosta [Ann. of Probability 3 (1975), 865-875], who proved a similar result for $\alpha \in(1,2)$, and the author [Proc. Amer. Math. Soc. 63 (1977), 306-312], who proved it for $\alpha \in[1,2)$. Further, it provides a complete affirmative solution to the question, raised by $J$. Kuelbs and V. Mandrekar [Studia Math. 50 (1974), 149-162], of whether the supp. of a symmetric stable probability measure of index $\alpha \in(0,1]$ on a separable Hilbert space $H$ is a subspace of $H$.
\end{abstract}

1. Introduction. The main purpose of this paper is to prove that the topological support (supp.) of a symmetric $\tau$-regular infinitely divisible (i.d.) (respectively, stable of any index $\alpha \in(0,2)$ ) probability measure (p.m.) on a locally convex topological vector space (LCTVS) $E$ is a (closed) subgroup (respectively, a subspace) of $E$ (Theorems 1,2). The part regarding the support of a stable p.m. of this result completes a result of De-Acosta [4], who proved a similar result for $\alpha \in(1,2)$, and the author [6], who proved it for $\alpha \in[1,2)$. It also settles a question raised by Kuelbs and Mandrekar [5].

2. Preliminaries. All topological spaces considered are assumed Hausdorff and all measures are defined on the Borel $\sigma$-algebra (the $\sigma$-algebra generated by the class of open sets) of the topological space. All vector spaces considered are over the real field $R$. If $A$ is a subset of a topological space $E$, then $\bar{A}$ will denote, throughout, the $E$-closure of $A$.

Definition 1. Let $\mu$ be a finite measure on a topological space $E$. Then $\mu$ is called $\tau$-regular if $\mu\left(\cup_{\alpha} O_{\alpha}\right)=\lim _{\alpha} \mu\left(O_{\alpha}\right)$ for every increasing net $\left\{O_{\alpha}\right\}$ of open sets of $E$. The unique smallest closed set with full $\mu$-measure (if it exists) is called the supp. of $\mu$. It is easy to show that whenever the supp. of a finite measure exists, then it is the set of those points of the space, every open neighborhood (nbd.) of which has positive $\mu$-measure. Also, if $\mu$ is $\tau$-regular

Presented to the Society, October 30, 1976 under the title On the subgroup (res. sub-space) property of the support of symmetric infinitely divisible (res. stable) probability measures on LCTVS; received by the editors June 28, 1976 and, in revised form, April 15, 1977.

AMS (MOS) subject classifications (1970). Primary 60B05, 28A40; Secondary 46G99, 60B15.

Key words and phrases. Locally convex topological vector space, infinitely divisible and stable probability measures, Gaussian probability measure, topological support.

(c) American Mathematical Society 1977 
then the supp. of $\mu$ always exists. If $\mu$ denotes a finite measure on a topological space, then, throughout, $S_{\mu}$ will denote the supp. of $\mu$.

Definition 2. Let $\mu$ and $\nu$ be two $\tau$-regular finite measures on a LCTVS $E$; then, according to [1] and [2], the convolution of $\mu$ and $\nu$, denoted by $\mu * \nu$, is defined by

$$
\mu * \nu(A)=\int_{E} \mu(A-x) \nu(d x)=\int_{E} \nu(A-x) \mu(d x),
$$

for every Borel set $A$.

Definition 3. Let $\mu$ be a $\tau$-regular p.m. on a LCTVS $E$; then $\mu$ is called i.d. if for each $n=1,2, \ldots$, there exists a $\tau$-regular p.m. $\lambda_{n}$ on $E$ such that $\mu={ }^{*} \lambda_{n}^{n}, \lambda_{n}$ convoluted with itself $n$-times. $\mu$ is called stable if for each $a, b \in R, \mathrm{a}>0, \mathrm{~b}>0$ there exist $c>0$ and $x \in E$ such that $T_{a} \mu * T_{b} \mu=$ $T_{c} \mu * \delta_{x}$, where (for a given $\left.r \in R, r \neq 0\right) T_{r} \mu(A)=\mu\left(r^{-1} A\right)$ for every Borel set $A$, and $\delta_{x}$ denotes the degenerate p.m. at $x$.

Since $\tau$-regularity is implicit in the definitions of i.d. and stable p.measures, in the sequel, whenever we talk of i.d. or stable p.m., it will be implicitly assumed that it is $\tau$-regular. For more information on stable and i.d. p.measures see [3] and [9], respectively. A result from [3] which is relevant in the sequel is that for a given stable p.m. $\mu$ on a LCTVS $E$ there exists a unique $\alpha \in(0,2]$ such that $c$ in the above definition is equal to $\left(a^{\alpha}+b^{\alpha}\right)^{1 / \alpha}$; this $\alpha$ is referred to as the index of $\mu$ (note that if $\alpha=2$, then $\mu$ is Gaussian, i.e. every continuous linear functional on $E$ is a real Guassian (possibly degenerate) random variable).

Definition 4. A p.m. $\mu$ on a separable normed linear space $B$ is called compound Poisson if

$$
\mu \equiv e(\nu)=e^{-\nu(B)} \sum_{k=0}^{\infty} \frac{{ }^{*} \nu^{k}}{k !},
$$

where $\nu$ is a finite measure and ${ }^{*} \nu^{0}=\delta_{0}$.

3. Statement and proof of results. We will prove the following two theorems:

THEOREM 1. Let $\mu$ be a symmetric i.d.p.m. on a LCTVS E; then $S_{\mu}$ is a (closed) subgroup (under addition) of $E$.

THEOREM 2. Let $\mu$ be a symmetric stable p.m. of any index $\alpha \in(0,2)$ on a LCTVS E; then $S_{\mu}$ is a (closed) subspace of $E$.

For the proofs of these theorems we will need the following results.

LEMMA 1 [2, P. 37]. Let $\mu$ and $\nu$ be two p. measures on a separable Banach space. Then $S_{\mu * \nu}=\overline{S_{\mu}+S_{\nu}}$.

LEMMA 2 (TORTRAT [9]). If $\mu$ is a symmetric i.d.p.m. on a separable Banach space $B$, then $\mu=\nu * \rho$, with $\rho$ centered Gaussian and $\nu=\lim _{n} \nu_{1} * \cdots * \nu_{n}$, where each $\nu_{j}$ is symmetric compound Poisson, and the limit is in the sense of 
weak convergence of $p$. measures. Further, for every $n, \nu=\nu_{1} * \cdots * \nu_{n} * \rho_{n}$, for some symmetric i.d.p.m. $\rho_{n}$.

Proof. In view of Theorems II and V of [9], it remains to prove that the factors are symmetric and that $\nu$ is the limit of $\left\{\nu_{1} * \ldots * \nu_{n}\right\}$ rather than being the limit of certain shifts of it. Since $\mu$ is symmetric i.d., $\mu={ }^{*} \mu_{1}^{2}$, where $\mu_{1}$ is symmetric i.d. From the cited result of [9], $\mu_{1}=\lambda * \sigma$, with $\sigma$ Gaussian and $\lambda=\lim _{n} \lambda_{1} * \cdots * \lambda_{n} * \delta_{x_{n}}$, where the $\lambda_{n}$ 's are compound Poisson and $x_{n} \in B$. Also

$$
T_{-1} \mu_{1}=T_{-1} \lambda * T_{-1} \sigma \quad \text { and } T_{-1} \lambda=\lim _{n} T_{-1} \lambda_{1} * \cdots * T_{-1} \lambda_{n} * \delta_{-x_{n}} .
$$

Hence, by [3],

$$
\nu \equiv \lambda * T_{-1} \lambda=\lim _{n}\left(\lambda_{1} * T_{-1} \lambda_{1}\right) * \cdots *\left(\lambda_{n} * T_{-1} \lambda_{n}\right)
$$

Hence taking $\nu_{j}=\lambda_{j} * T_{-1} \lambda_{j}, j=1,2, \ldots$, and $\rho=\sigma * T_{-1} \sigma$, we get the first two conclusions of the lemma; the last conclusion also follows from similar arguments.

LEMMA 3. Let $\mu$ be a symmetric i.d. p.m. on a separable Banach space $B$. Then $0 \in S_{\mu}$.

Proof. As in the proof above, $\mu={ }^{*} \mu_{1}^{2}$, where $\mu_{1}$ is symmetric i.d. Hence, $S_{\mu_{1}}=-S_{\mu_{1}}$ and $S_{\mu}=\overline{S_{\mu_{1}} \pm S_{\mu_{1}}}$ (Lemma 1), implying $0 \in S_{\mu}$.

LEMMA 4. Let $\nu$ be a finite measure on a separable Banach space $B$ and $\mu=e(\nu)$. Then $S_{\mu}=\overline{\bigcup_{k} k S_{\nu}}$, where $k S_{\nu}$ is $n$-fold sum of $S_{\nu}$. In particular, $S_{\mu}$ is a subsemigroup; further, if $\mu$ is symmetric then $S_{\mu}$ is a subgroup.

Proof. Since $S_{{ }_{\nu}} k=\overline{k S_{\nu}}$ (Lemma 1), $S_{\mu} \supseteq \overline{\cup_{k} k S_{v}}$. On the other hand,

$$
\mu\left(\overline{\bigcup_{k} k S_{\nu}}\right)=\sum_{k} \frac{{ }^{*} \nu^{k}\left(k S_{\nu}\right) e^{-\nu(E)}}{k !}=\sum_{k} \frac{{ }^{*} \nu^{k}(E) e^{-\nu(E)}}{k !}=1,
$$

implying $\overline{\cup_{k} k S_{\nu}} \supseteq S_{\mu}$, completing the proof.

Proof of TheOREM 1. First we show that one needs to prove the result only in a separable Banach space. Let $\hat{E}$ be the completion of $E$ and $u$ be the natural injection of $E$ into $\hat{E}$. Let $\nu=\mu \circ u^{-1}$; then one notes that $\nu$ is symmetric, $\tau$-regular and i.d. Since $\hat{E}$ is a complete LCTVS, $\hat{E}=$ proj $\lim g_{\alpha, \beta} E_{\alpha}$, the projective limit of a projective system $\left\{E_{\alpha}, g_{\alpha, \beta}, \alpha<\beta\right\}$ of Banach spaces relative to some index set $I[8$, p. 53]. For each $n=$ $1,2, \ldots$, let $\nu_{n}$ be a $\tau$-regular p.m. on $\hat{E}$ such that $\nu={ }^{*} \nu_{n}^{n}$. Fix $\alpha$, and let $\lambda_{\alpha}=\nu \circ \pi_{\alpha}^{-1}$, and $\lambda_{\alpha, n}=\nu_{n} \circ \pi_{\alpha}^{-1}, n=1,2, \ldots$, where $\pi_{\alpha}$ is the natural projection of $\hat{E}$ into $E_{\alpha}$. One notes that $\lambda_{\alpha}$ and $\lambda_{\alpha, n}, n=1,2, \ldots$, are $\tau$-regular; therefore, by [7, Lemma 2], $S_{\lambda_{a}}$ and $S_{\lambda_{a, n}}, n=1,2, \ldots$, are separable subsets of $E_{\alpha}$. Let $B_{\alpha}$ be the $E_{\alpha}$-closure of the linear hull of the set $S_{\lambda_{\alpha}} \cup\left(\cup_{n=1}^{\infty} S_{\lambda_{\alpha, n}}\right)$; then $B_{\alpha}$ is a separable Banach subspace of $E_{\alpha}$. Further, since $\lambda_{\alpha}\left(B_{\alpha}\right)=\lambda_{\alpha, n}\left(B_{\alpha}\right)=1$ and $\lambda_{\alpha}={ }^{*} \lambda_{\alpha, n}^{n}$ for all $n=1,2, \ldots, \lambda_{\alpha}$ restricted to $B_{\alpha}$ is i.d. and clearly symmetric. Therefore, if we can show that $S_{\lambda_{\alpha}}$ is a 
(closed) subgroup of $B_{\alpha}$, then by [7, Proposition 1] $S_{\nu}$ would be a subgroup of $E$. Then since, by [7, Lemma 1], $S_{\mu}=S_{\nu} \cap E$, it will follow that $S_{\mu}$ is a subgroup.

Thus we assume that $E$ is a separable Banach space and prove the result in the Banach space setting. By Lemma $2, \mu=\nu * \rho$, where $\rho$ is centered Gaussian and $\nu=\lim _{n} \nu_{1} * \cdots * \nu_{n}$ with each $\nu_{j}$ symmetric compound Poisson. Thus, since $S_{\rho}$ is known to be a subspace [6], [7] and, since by Lemma $1, S_{\mu}=\overline{S_{\nu}+S_{\rho}}$, the proof will be complete if we can show that $S_{\nu}$ is a subgroup.

Let $\lambda_{n}=\nu_{1} * \cdots * \nu_{n}$; then each $\lambda_{j}$ is symmetric and compound Poisson. Therefore, by Lemma 4, $S_{\lambda_{n}}$ is a subgroup. Thus, since $S_{\lambda_{n}} \uparrow$ as $n \uparrow$ (Lemma 1, again), it follows that $S \equiv \cup_{n=1}^{\infty} S_{\lambda_{j}}$ and, hence, $\bar{S}$ is a subgroup. We will complete the proof by showing that $\bar{S}=S_{v}$. Since $\bar{S}$ is closed and $\lim _{n} \lambda_{n}=$ $\nu, \nu(\bar{S}) \geqslant \varlimsup \bar{l} \lambda_{n}(\bar{S})=\varlimsup{ }_{n}\left(S_{\lambda_{n}}\right)=1$. Thus $\bar{S} \supseteq S_{\nu}$. From the second part of Lemma 2, we have, for every $n, \nu=\lambda_{n} * \rho_{n}$, where $\rho_{n}$ is a symmetric i.d. p.m.; therefore, since by Lemma 3, $0 \in S_{\rho_{n}}$, we have, by Lemma $1, S_{\nu} \supseteq S_{\lambda_{n}}$ for all $n$. Consequently, $S_{v} \supseteq \bar{S}$, completing the proof.

Proof of THEOREM 2. This follows from Theorem 1, the fact that stable $p$. measures on a LCTVS are i.d. (Lemma 3.2 of [6]) and a result due to the author (see the proof of Theorem 3.1 of [6]), which asserts that for any symmetric stable p.m. $\mu$ on a LCTVS $E, a S_{\mu} \subseteq S_{\mu}$ for all real $a$ with $|a| \leqslant 1$. We should point out, however, that even though in the statement of Theorem 3.1 of [6] it is assumed that $1 \leqslant \alpha \leqslant 2$, a careful look at the proof of this theorem given in [6] shows that this hypothesis is not needed to conclude that $a S_{\mu} \subseteq S_{\mu}$ for $|a| \leqslant 1$.

ACKNOWLEDGEMENT. The author is grateful to the referee for his critical reading of the manuscript and for a suggestion which slightly shortened the proof of Lemma 4.

\section{REFERENCES}

1. I. Csiszar, Some problems concerning measures on topological spaces and convolutions of measures on topological groups, Les Probabilites sur les Structures Algébriques (Colloques Internat. de CNRS, Paris, 1970), Clermont-Ferrand, pp. 75-79.

2. On the weak ${ }^{*}$ convergence of convolution in a convolution algebra over an arbitrary group, Studia Sci. Math. Hungar. 6 (1971), $27-40$.

3. I. Csiszar and B. S. Rajput, A convergence of types theorem for probability measures on TVS with applications to stable laws, Z. Wahrscheinlichkeitstheorie und verw. Gebiete 36 (1976), 1-7.

4. A. De-Acosta, Stable measures and seminorms, Ann. of Probability 3 (1975), 865-875.

5. J. Kuelbs and V. Mandrekar, Domains of attractions of stable measures on Hilbert space, Studia. Math. 50 (1974), 149-162.

6. B. S. Rajput, On the support of certain symmetric stable probability measures on TVS, Proc. Amer. Math. Soc. 63 (1977), 306-312.

7. B. S. Rajput and N. N. Vakhania, On the support of Gaussian probability measures on LCTVS, Fourth Internat. Sympos. on Multivariable Analysis (P. R. Krishnaiah, editor), NorthHolland, New York, 1977, pp. 297-309.

8. H. H. Schaefer, Topological vector spaces, 3rd printing, Springer, New York, 1970.

9. A. Tortrat, Structure des lois indéfiniment divisibles dans un espace vectoriel topologique, Lecture Notes in Math., vol. 31, Springer-Verlag, New York, 1967, pp. 299-327. 